\title{
Cholesterol granuloma in the wall of a mandibular dentigerous cyst: a rare case report
}

\author{
JH Lee ${ }^{\text {, }}$ MS Alrashdan ${ }^{2}$, KM Ahn ${ }^{1}$, MH Kang ${ }^{1}$, SP Hong ${ }^{3}$, SM Kim ${ }^{1}$ \\ ${ }^{1}$ DDS, PhD. Department of Oral and Maxillofacial Surgery, School of Dentistry, Seoul National University \\ ${ }^{2}$ BDS, MSD. Department of Oral and Maxillofacial Surgery, School of Dentistry, Seoul National University \\ ${ }^{3}$ DDS, PhD. Department of Oral Pathology, School of Dentistry, Seoul National University
}

\author{
Correspondence: \\ Soung-Min Kim \\ Address: 275-1, Yeongeon-Dong, Jongno-Gu, \\ Department of Oral and Maxillofacial Surgery, Seoul National University Dental Hospital, \\ Seoul 110-768, Rep. of Korea. \\ e-mail:smin5@snu.ac.kr
}

Received: $25 / 01 / 2009$

Accepted: 29/03/2010

Lee JH, Alrashdan MS, Ahn KM, Kang MH, Hong SP, Kim SM. Cholesterol granuloma in the wall of a mandibular dentigerous cyst: a rare case report. J Clin Exp Dent. 2010;2(2):e88-90.

http://www.medicinaoral.com/odo/volumenes/v2i2/jcedv2i2p88.pdf

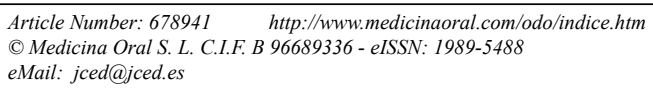

\begin{abstract}
Cholesterol granuloma is an inflammatory reaction to cholesterol crystals deposition. It may develop in a variety of sites including the middle ear, mastoid process or even paranasal sinuses. Very few cases of cholesterol granuloma occurring in the jaws were reported. This report presents a rare case of cholesterol granuloma that developed in the wall of a large mandibular dentigerous cyst. The condition was treated with hemimandibulectomy followed by reconstruction with a free fibular flap.
\end{abstract}

Key words: Cholesterol granuloma, mandible, dentigerous cyst.

*Note: This report was presented during the 47th Congress of the Korean Association of Maxillofacial Plastic and Reconstructive Surgeons. April 2009, Seoul, Rep. of Korea.

*This report was supported by Ministry of Knowledge and Economy(10033290) 


\section{Introduction}

Cholesterol granuloma represents an inflammatory foreign body reaction in response to cholesterol crystals. It usually develops in association with chronic middle ear diseases and affects the mastoid antrum and air cells within the temporal bone. However, it can rarely develop in the paranasal sinuses $(1,2)$.

Only very few cases of cholesterol granuloma occuring in jaws were reported in the English literature. Here we present an extremely rare case of cholesterol granuloma within a mandibular dentigerous cyst wall.

\section{Case report}

A 68 years old male patient was referred to Seoul National University Dental Hospital, complaining of a chronic swelling in the left cheek area associated with cheek biting, of 2 years duration.
Clinical examination revealed thickening of the left buccal mucosa with traumatic ulceration due to chronic cheek biting.

Radiographic examination showed a large multilocular radiolucency surrounding a horizontally impacted third molar in the left side of the mandible (Fig. 1). The lesion was managed by hemimandibulectomy followed by reconstruction using a fibular free flap.

Mass gross specimen examination of the resected hemimandible from the lower right central incisor to the coronoid process showed cortical bone thinning, with 2 distinct parts. An anterior part containing a mud-like, grayish brown granulomatous material and an empty posterior part containing the left mandibular third molar. The size of the resected mass was $10.5 \times 7.5 \times 3.5 \mathrm{~cm}$ (Fig. 2).

Histologic examination of the resected tissue by light

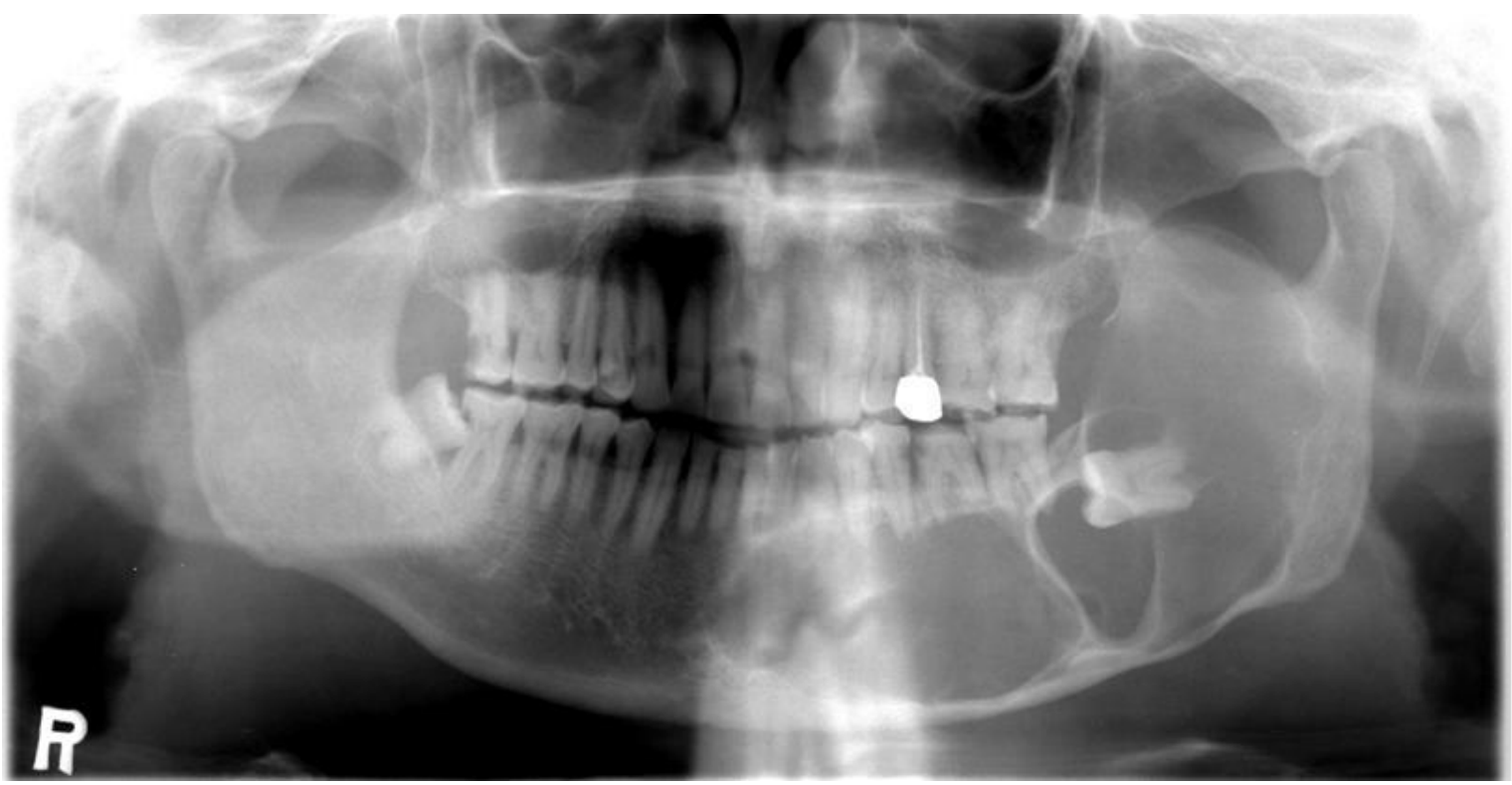

Fig. 1. Preoperative panoramic radiograph showing a huge multilocular radiolucency, involving an impacted mandibular third molar, extending from the left sigmoid notch to the contralateral symphisis area. Root resorption is seen in teeth 3.5, 3.6.

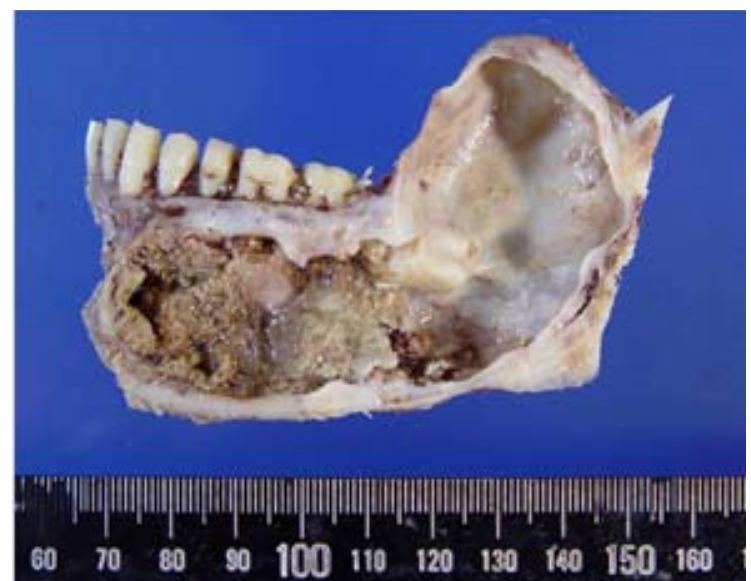

Fig. 2. Gross specimen of the resected hemimandible, showing 2 distinct areas. The anterior part contains grayish, mud-like granulomatous material while the posterior part is empty.

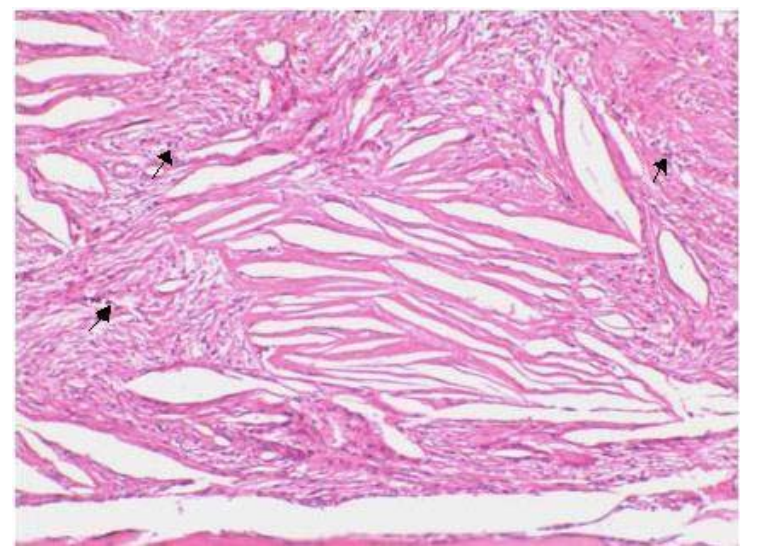

Fig.3. Photomicrographs showing histopathological features of the cholesterol granuloma; large number of white spindle-shaped clefts representing cholesterol clefts, with lymphocytes(arrows) infiltration. Noepithelialcellsareseen.(Hematoxylinandeosinstain, X100). 
microscopy revealed that the posterior part of the lesion contained 3-5 layers of flattened non-keartinized epithelial cells surrounded by a thin fibrous capsule with no inflammatory cells. The anterior part, however, was completely occupied by cholesterol granulomatous tissue with heavy lymphocyte infiltration between the cholesterol clefts within a fibrous connective tissue stroma. No presence of epithelial cells was evident (Fig. 3). The presence of hemosiderin in the anterior part may be an evidence of previous hemorrhage. The final diagnosis of the resected lesion was a dentigerous cyst with cholesterol granuloma occupying its anterior part.

\section{Discussion}

Differential diagnosis of multilocular radiolucencies within the jaws includes, but is not limited to, ameloblastomas, dentigerous cysts and odontogenic keratocysts. Dentigerous cysts are the most common developmental odontogenic cysts in the jaws. Although they are not true multilocular lesions, they can grow to a considerable size and give such an impression on radiographs due to the presence of bone trabeculation within the cystic cavity. Most commonly they develop in association with impacted mandibular third molars, less frequently with impacted maxillary canines or other teeth. Usually they are asymptomatic unless infected or growing to a considerable degree causing bony expansion and asymmetry (3).

Cholesterol granuloma describes a large collection of longitudinal clefts representing sites of cholesterol crystals that dissolved during processing, with surrounding foreign body giant cells and macrophages filled with hemosiderin and embedded in fibrous granulation tissue (4). It can develop in a variety of body areas such as: the middle ear (most common site), mastoid process testis, lungs, apex of temporal bone pyramid and paranasal sinuses (5).

Unlike cholesterol granuloma, development of ameloblastoma or even squamous cell carcinoma within dentigerous cysts have been previously reported $(6,7)$.

The pathogenesis of cholesterol granuloma is still unclear, but in the middle ear it is seems to be related to obstruction of ear drainage. Disturbance of air drainage would cause negative pressure within the air cavity secondary to absorption of air into mucosa. As a result, mucosal edema and hemorrhage develop. Hematoma from the mucosal bleeding would not be absorbed, resulting in its conversion to cholesterol crystals. The deposited cholesterol crystals act as an irritant which attracts foreign body giant cells and causes fibrosis (2).Based on this mechanism, cholesterol granuloma is not expected to occur in the mandible due to the absence of intrabony cavities or drainage pathways.

A recent study by Yamazaki et al. (8) suggests that perlecan (a basement membrane heparan sulfate proteo- glycan) which is present abundantly in the cyst wall of immature granulation tissue might be related to the development of cholesterol granulomas.

To the best of our knowledge, the case reported by Hirschberg et al. (9) is the only case of cholesterol granuloma reported in the mandible in English literarure. Kaffe et al. (10) reported another case of cholesterol granuloma in a maxillary odontoma. This is the first reported case of cholesterol granuloma within a mandibular odotogenic cyst.

\section{References}

1. Leon ME, Chavez C, Fyfe B, Nagorsky MJ, Garcia FU. Cholesterol granuloma of the maxillary sinus. Arch Pathol Lab Med. 2002;126:217-9.

2. Ochiai H, Yamakawa Y, Fukushima T, Nakano S, Wakisaka S. Large cholesterol granuloma arising from the frontal sinus--case report. Neurol Med Chir (Tokyo). 2001;41:283-7.

3. Mosqueda-Taylor A, Irigoyen-Camacho ME, Diaz-Franco MA, Torres-Tejero MA. Odontogenic cysts. Analysis of 856 cases. Med Oral. 2002;7:89-96.

4. Chao TK. Cholesterol granuloma of the maxillary sinus. Eur Arch Otorhinolaryngol. 2006;263:592-7.

5. Almada CB, Fonseca DR, Vanzillotta RR, Pires FR. Cholesterol granuloma of the maxillary sinus. Braz Dent J. 2008;19:171-4.

6. Gulbranson SH, Wolfrey JD, Raines JM, McNally BP. Squamous cell carcinoma arising in a dentigerous cyst in a 16-month-old girl. Otolaryngol Head Neck Surg. $2002 ; 127: 463-4$.

7. Houston GD.Oral Pathology.Ameloblastoma arising in a dentigerous cyst.J Okla Dent Assoc. 2007;98:28-9.

8. Yamazaki M,Cheng J, Hao N,Takagi R, Jimi S, Itabe H, et al. Basement membrane-type heparan sulfate proteoglycan (perlecan) and low -density lipoprotein(LDL) are co-localized in granulation tissues: a possible pathogenesis of cholesterol granulomas in jaw cysts. J Oral pathol Med. 2004; 33:177-84

9. Hirschberg A, Dayan D, Buchner A, Freedman A. Cholesterol granuloma of the jaws. Int J Oral Maxillofac Surg.1988; 17:230-1

10. Kaffe I, Littner MM, Buchner A, Friedman M. Cholesterol granuloma embedded in an odontoma of the maxilla. J Oral Maxillofac Surg. 1984; 42:319-22. 